\title{
MicroRNA-133a inhibits proliferation and invasion, and induces apoptosis in gastric carcinoma cells via targeting fascin actin-bundling protein 1
}

\author{
CHEN LAI, ZIHUA CHEN and RUIXIN LI
}

Department of General Surgery, Xiangya Hospital of Central South University, Changsha, Hunan 410008, P.R. China

Received June 16, 2014; Accepted March 4, 2015

DOI: $10.3892 / \mathrm{mmr} .2015 .3545$

\begin{abstract}
Fascin actin-bundling protein 1 (FSCN1) is associated with tumor progression. In addition, deregulation of the expression of FSCN1 has been observed in certain types of cancer. However, the detailed role of FSCN1 in gastric cancer remains to be elucidated. In the present study, downregulation of microRNA (miR)-133a and upregulation of FSCN1 were both observed in gastric cancer tissues and cell lines. Functional studies have revealed that miR-133a is able to bind to the 3'-untranslated region of FSCN1 mRNA, and overexpression of miR-133a causes downregulation of FSCN1 expression, while downregulation of miR-133a leads to an increased FSCN1 expression in gastric cancer cells. Furthermore, overexpression of miR-133a inhibited proliferation and invasion, but promoted apoptosis of gastric cancer cells, which may be reversed by upregulation of FSCN1. By contrast, downregulation of miR-133a enhanced proliferation and invasion, but suppressed apoptosis in gastric cancer cells. In conclusion, the anti-oncogenic activity of miR-133a may involve the inhibition of the target gene FSCN1. The present study suggested that miR-133a may be a potential therapeutic target in the treatment of gastric cancer.
\end{abstract}

\section{Introduction}

Gastric cancer is the fourth most common type of cancer and the second leading cause of cancer-associated mortality globally (1). Although the incidence and rates of mortality have decreased over the past 20 years, patients with gastric cancer are generally diagnosed at an advanced stage and the prognosis is often poor (2). Furthermore, the molecular mechanisms involved in the development and progression of gastric cancer remain to be elucidated $(3,4)$.

Correspondence to: Professor Zihua Chen, Department of General Surgery, Xiangya Hospital of Central South University, 87 Xiangya Road, Changsha, Hunan 410008, P.R. China

E-mail: csuchenzihua@163.com

Key words: gastric carcinoma, fascin actin-bundling protein 1, microRNA-133a, proliferation, apoptosis, invasion
The deregulation of oncogenes or tumor suppressors, including microRNAs (miRNAs/miRs), has been observed to be involved in the development and progression of gastric cancer $(5,6)$. As a type of small noncoding RNA, miRNAs are able to modulate multiple cellular processes by affecting the post-transcriptional processes regulating gene expression, which are able to promote or inhibit the development and progression of human malignancies (7). It has been widely-accepted that the upregulation or downregulation of miRNAs is able to affect the expression of various proteins and therefore is involved in tumorigenesis through mediating proliferation and invasion (8).

Aberrant expression of certain miRNAs, including miR-133a, has been found to be correlated with the development, progression and prognosis of gastric cancer. In addition, miR-133a generally acts as a tumor suppressor in various types of cancer, including gastric cancer (9), non-small cell lung cancer (10), ovarian cancer (11), osteosarcoma (12) and esophageal squamous cell carcinoma (12). More recently, Qiu et al (9) reported that miR-133a is able to inhibit proliferation, migration, invasion and cell cycle progression via targeting transcription factor specificity protein 1 in gastric cancer cells. As miRNAs are able to target various mRNAs, other oncogenes may also be involved in miR-133a-mediated cell proliferation and invasion in gastric cancer cells (5).

The present study aimed to examine the roles of miR-133a and FSCN1 in the regulation of the malignant phenotypes of gastric cancer cells. In addition, the association between miR-133a and FSCN was investigated.

\section{Materials and methods}

Reagents. Dulbecco's modified Eagle's medium (DMEM), fetal bovine serum (FBS), TRIzol reagent, Lipofectamine 2000, SYBR green assay kit, miRNA reverse transcription kit, and all the miRNA mimics and the inhibitor were purchased from Invitrogen Life Technologies (Carlsbad, CA, USA). The bicinchoninic acid (BCA) protein assay kit and an enhanced chemiluminescence (ECL) kit were purchased from Pierce Biotechnology, Inc. (Rockford, IL, USA). MTT was purchased from Biosharp (Hefei, China). Hairpin-it ${ }^{\mathrm{TM}}$ miRNAs qPCR quantitation kit and U6 small nuclear RNA were purchased from Gene-Pharma (Shanghai, China). PsiCHECK ${ }^{\text {тм }} 2$ vector was purchased from Promega Corporation (Madison, WI, 
USA). A quick-change site-directed mutagenesis kit was purchased from Stratagene (La Jolla, CA, USA). Mouse anti-FSCN1 monoclonal antibody (cat. no. ab49815) and mouse anti-GAPDH (cat. no. ab8245) monoclonal antibody were purchased from Abcam (Cambridge, UK). An Annexin V-fluorescein isothiocyanate (FITC) apoptosis detection kit was purchased from BD Pharmingen (San Diego, CA, USA) A Transwell chamber was obtained from Corning Inc. (Corning, NY, USA).

Tissue specimen collection. The present study was approved by the Ethical Committee of Central South University (Changsha, China). Informed consent was obtained from the patients. A total of 18 gastric carcinoma tissues and normal gastric tissues were obtained from the Department of General Surgery, Xiangya Hospital of Central South University (Changsha, China) between November 2012 and June 2013 The gastric carcinoma tissue samples were obtained during surgical resection and confirmed by pathological examination. Normal gastric tissue was obtained during surgery as a result of gastrorrhagia and confirmed by pathological examination. The tissue samples were immediately frozen in liquid nitrogen following surgical removal.

Cell culture. HGC-27, GC7901 and AGS human gastric carcinoma cells, and GES-1 normal gastric mucosa epithelial cells were obtained from the Cell Bank of Central South University, and cultured in DMEM with $10 \% \mathrm{FBS}$ at $37^{\circ} \mathrm{C}$ in a humidified incubator containing $5 \% \mathrm{CO}_{2}$.

Reverse transcription-quantitative polymerase chain reaction $(R T-q P C R)$ assay. According to the manufacturer's instructions, total RNA was extracted using TRIzol reagent. Subsequently, an miRNA reverse transcription kit was used to convert RNA into cDNA. Subsequently, the expression levels of miRNAs were evaluated using the Hairpin-it ${ }^{\mathrm{TM}}$ miRNAs qPCR quantitation kit, according to the manufacturer's instructions. The relative expression of the miRNAs were analyzed using the $2^{-\Delta \Delta \mathrm{Ct}}$ method. The U6 small nuclear RNA was used for normalization. The expression of FSCN1 mRNA was detected using the SYBR green qPCR assay kit. Expression of GAPDH was used as an endogenous control. The specific primer sequences were as follows: Forward: 5'-CCAGGGTATGGACCTGTCTG-3' and reverse: 5'-GTGTGGGTACGGAAGGCAC-3' for FSCN1; and forward: 5'-GGAGCGAGATCCCTCCAAAAT-3' and reverse: 5'-GGCTGTTGTCATACTTCTCATGG-3' for GAPDH.

Western blotting. The tissues or cells were lysed in radioimmunoprecipitation assay buffer. The protein was quantified using a BCA protein assay kit. The proteins $(60 \mu \mathrm{g})$ were separated by $10 \%$ SDS-PAGE, and transferred onto a polyvinylidene difluoride (PVDF) membrane (Pierce, Rockford, IL, USA), which was then incubated with Tris-buffered saline with Tween 20 containing 5\% milk at room temperature for $3 \mathrm{~h}$. The PVDF membrane was then incubated with rabbit anti-FSCN1 and GAPDH primary antibodies, respectively, at room temperature for $3 \mathrm{~h}$. Following three washes with phosphate-buffered saline with Tween 20, the membrane was incubated with the rabbit anti-mouse secondary antibodies (cat. no. ab175743; Abcam) at room temperature for $40 \mathrm{~min}$. Chemiluminescence detection was performed using an ECL kit. The relative protein expression was analyzed using Image-Pro plus software 6.0 (Media Cybernetics, Rockville, $\mathrm{MD}, \mathrm{USA})$, presented as the density ratio versus GAPDH.

Transfection. In accordance with the manufacturer's instructions, transfection was performed using Lipofectamine 2000. For FSCN1 functional analysis, the cells were transfected with FSCN1-specific small interfering RNA or a pcDNA3.1-FSCN1 plasmid. For miR-133a functional analysis, AGS cells were transfected with the scrambled miRNA as a negative control, miR-133a mimics, or miR-133a inhibitor.

Dual luciferase reporter assay. A quick-change site-directed mutagenesis kit was used to generate a mutant type 3'-untranslated region (UTR) of FSCN1, according to the manufacturer's instructions. The wild or mutant type 3'-UTR of FSCN1 was inserted into the psiCHECK ${ }^{\mathrm{TM}} 2$ vector, respectively. After AGS cells were cultured to $\sim 70 \%$ confluence, they were transfected with psiCHECK ${ }^{\mathrm{TM}}$ 2-FSCN1-3'-UTR or psiCHECK ${ }^{\mathrm{TM}}$ 2-mutant FSCN1 -3'-UTR vector, with or without $100 \mathrm{nM}$ miR-133a mimics, respectively. After transfection for $48 \mathrm{~h}$, the luciferase activities were determined using an LD400 luminometer (Beckman Coulter, Fullerton, CA, USA). Renilla luciferase activity was normalized to firefly luciferase activity.

Cell proliferation analysis. MTT was used to perform cell proliferation analysis, according to the manufacturer's instructions. Briefly, for each group, $1 \times 10^{4}$ cells per well were plated in a 96-well plate, and incubated for $0,12,24$ or $48 \mathrm{~h}$ at $37^{\circ} \mathrm{C}$ and $5 \% \mathrm{CO}_{2}$. To assess cell proliferation, $10 \mu \mathrm{MTT}$ $(5 \mathrm{mg} / \mathrm{ml})$ was added to each well and then incubated for $4 \mathrm{~h}$ at $37^{\circ} \mathrm{C}$ and $5 \% \mathrm{CO}_{2}$. The supernatant was removed, and $100 \mu \mathrm{l}$ dimethyl sulfoxide was added to dissolve the precipitate. The absorbance was detected at $492 \mathrm{~nm}$ with a Microplate Reader (Bio-Rad Laboratories, Inc., Hercules, CA, USA).

Apoptosis analysis. The Annexin V-FITC apoptosis detection kit was used to perform cell apoptosis analysis, according to the manufacturer's instructions. Briefly, cells were resuspended in binding buffer, then $2.5 \mu \mathrm{l}$ Annexin $\mathrm{V}$ and $5 \mu \mathrm{l}$ propidium iodide were added. Following incubation for $15 \mathrm{~min}$ in the dark, $400 \mu 1$ binding buffer was added. Cell apoptosis was determined via flow cytometry (FACSCalibur C6, Becton-Dickinson, Franklin Lakes, NJ, USA).

Invasion assay. A cell suspension containing $5 \times 10^{5}$ cells $/ \mathrm{ml}$ was prepared in serum-free media. Subsequently, $300 \mu 1$ cell suspension was added into the upper chamber, and $500 \mu \mathrm{l}$ RPMI 1640 containing 10\% FBS was added into the lower chamber. Following incubation for 24 h, non-invading cells as well as the matrix gel on the interior of the inserts was removed using a cotton-tipped swab. Invasive cells on the lower surface of the membrane were stained with $0.1 \%$ crystal violet for $20 \mathrm{~min}$, then rinsed with water and air dried. A total of five fields were randomly selected and the cell number was counted under a microscope (CX22; Olympus, Tokyo, Japan). 
A

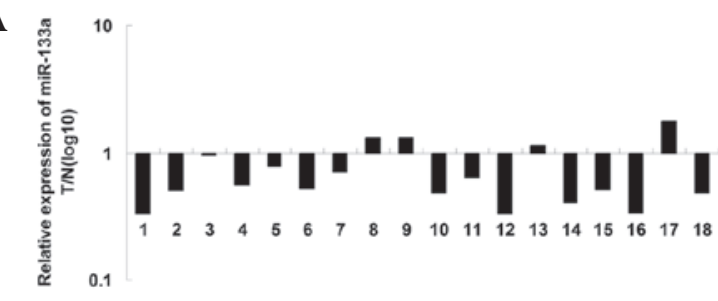

C

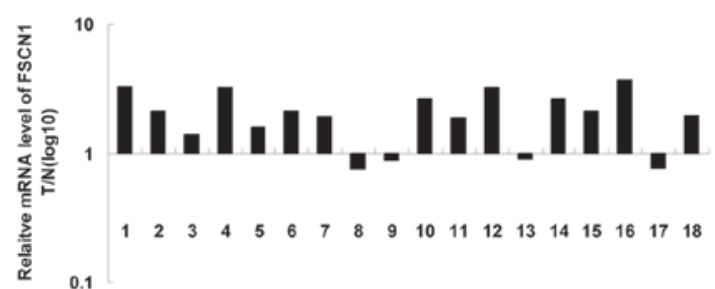

B

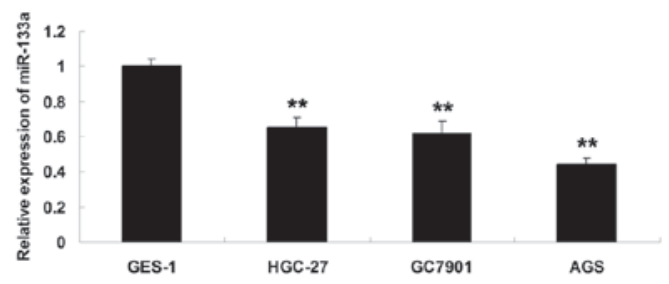

D

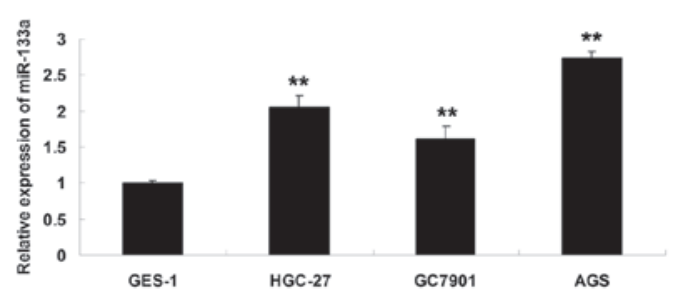

Figure 1. (A) RT-qPCR was performed to examine the relative expression of miR-133a in 18 gastric carcinoma tissues (Tumor) and their matched normal adjacent tissues (Normal). T/N: tumor vs. normal. (B) RT-qPCR was performed to examine the relative expression of miR-133a in three gastric carcinoma cell lines. Normal gastric mucosa epithelial GES-1 cells were used as a control. ${ }^{* *} \mathrm{P}<0.01$ vs. GES-1. (C) RT-qPCR was performed to examine the relative mRNA expression of FSCN1 in 18 gastric carcinoma tissues (Tumor) and their matched normal adjacent tissues (Normal). T/N: tumor vs. normal. (D) RT-qPCR was performed to examine the relative mRNA expression of FSCN1 in three gastric carcinoma cell lines, and GES-1 normal gastric mucosa epithelial cells were used as a control. GAPDH was used as an internal reference. ${ }^{* *} \mathrm{P}<0.01$ vs. GES-1. RT-qPCR, reverse transcription-quantitative polymerase chain reaction; miR, microRNA; FSCN1, fascin actin-bundling protein 1; UTR, untranslated region.

A

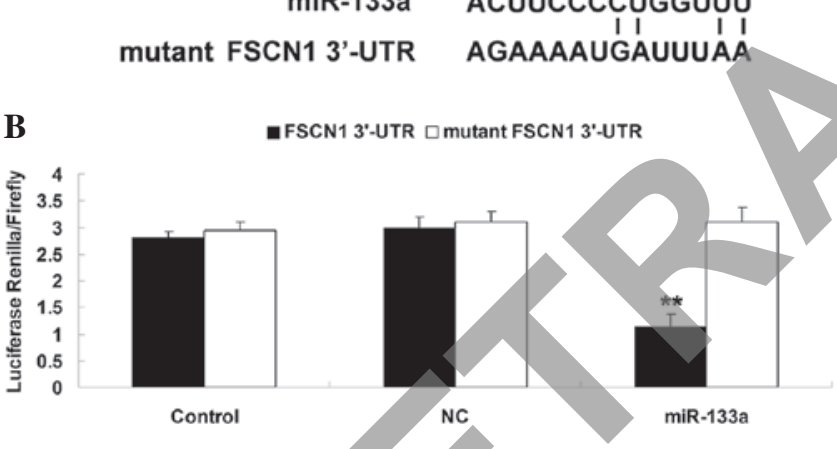

Figure 2. (A) Seed sequences of miR-133a in the wild type and mutant type 3'-UTR of FSCN1 are indicated. (B) The luciferase activity was notably decreased in AGS gastric carcinoma cells co-transfected with miR-133a mimics and the wild type 3'UTR of FSCN1, but unaltered in AGS cells co-transfected with miR-133a mimics and mutant FSCN1 3'UTR. Control: cells co-transfected with blank vector and wild type FSCN1 3'-UTR or mutant FSCN1 3'-UTR, respectively. ${ }^{* *} \mathrm{P}<0.01$ vs. control. miR, microRNA; FSCN1, fascin actin-bundling protein 1; UTR, untranslated region; NC, negative control.

Statistical analysis. The results are expressed as the mean \pm standard deviation of three independent experiments. Statistical analysis of the differences was performed using a one-way analysis of variance with SPSS 17.0 software (SPSS, Inc., Chicago, IL, USA). P<0.05 was considered to indicate a statistically significant difference.

\section{Results}

miR-133a is downregulated and FSCN1 is upregulated in gastric carcinoma tissues and cells. The expression level of miR-133a was initially examined in gastric carcinoma tissues and their matched normal adjacent tissues. As shown in Fig. 1A, the expression level of miR-133a in gastric carcinoma
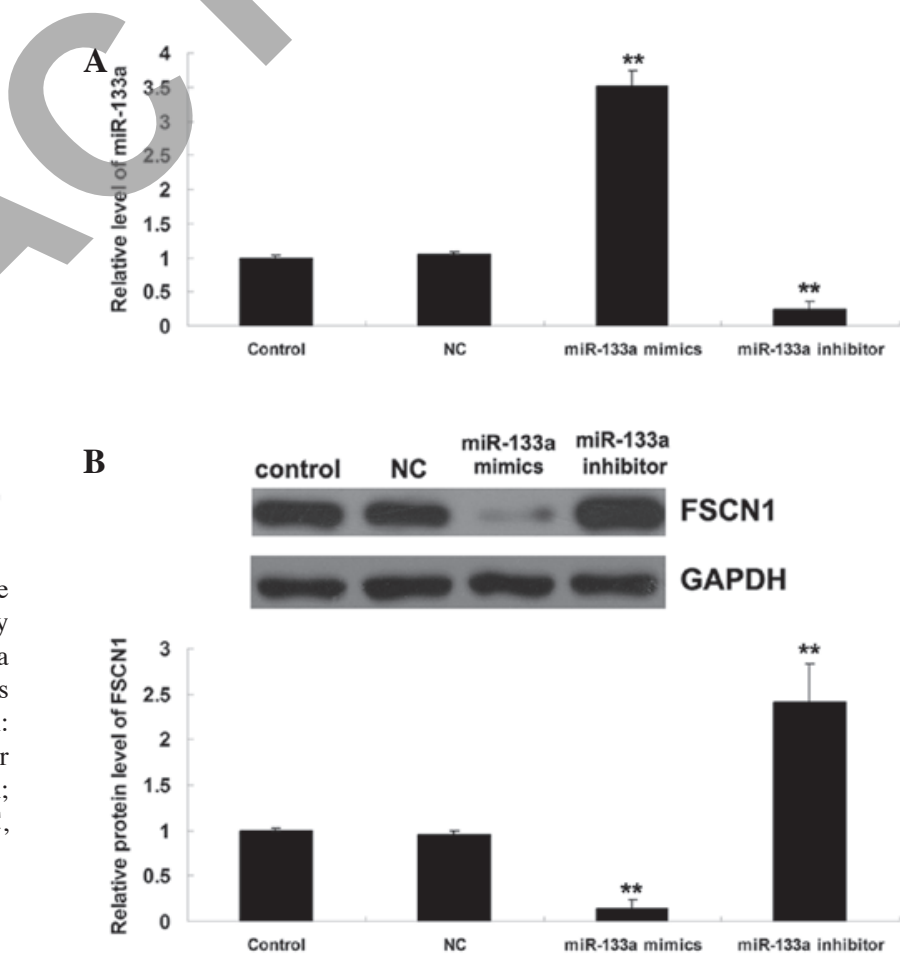

Figure 3. (A) Reverse transcription quantitative polymerase chain reaction was performed to determine the relative expression of miR-133a in AGS cells transfected with scramble miRNA (NC), miR-133a mimics and miR-133a inhibitor. (B) Western blotting was performed to examine the protein level of FSCN1 in AGS cells transfected with scramble miRNA (NC), miR-133a mimics and miR-133a inhibitor. Control: AGS cells without any transfection. ${ }^{* *} \mathrm{P}<0.01$ vs. control. miR, microRNA; FSCN1, fascin actin-bundling protein $1 ; \mathrm{NC}$, negative control.

tissues was significantly reduced. In addition, it was also downregulated in gastric carcinoma cell lines, when compared with normal gastric epithelial cells (Fig. 1B). Subsequently, the expression level of FSCN1 was determined using RT-qPCR. The present findings revealed that the mRNA level of FSCN1 


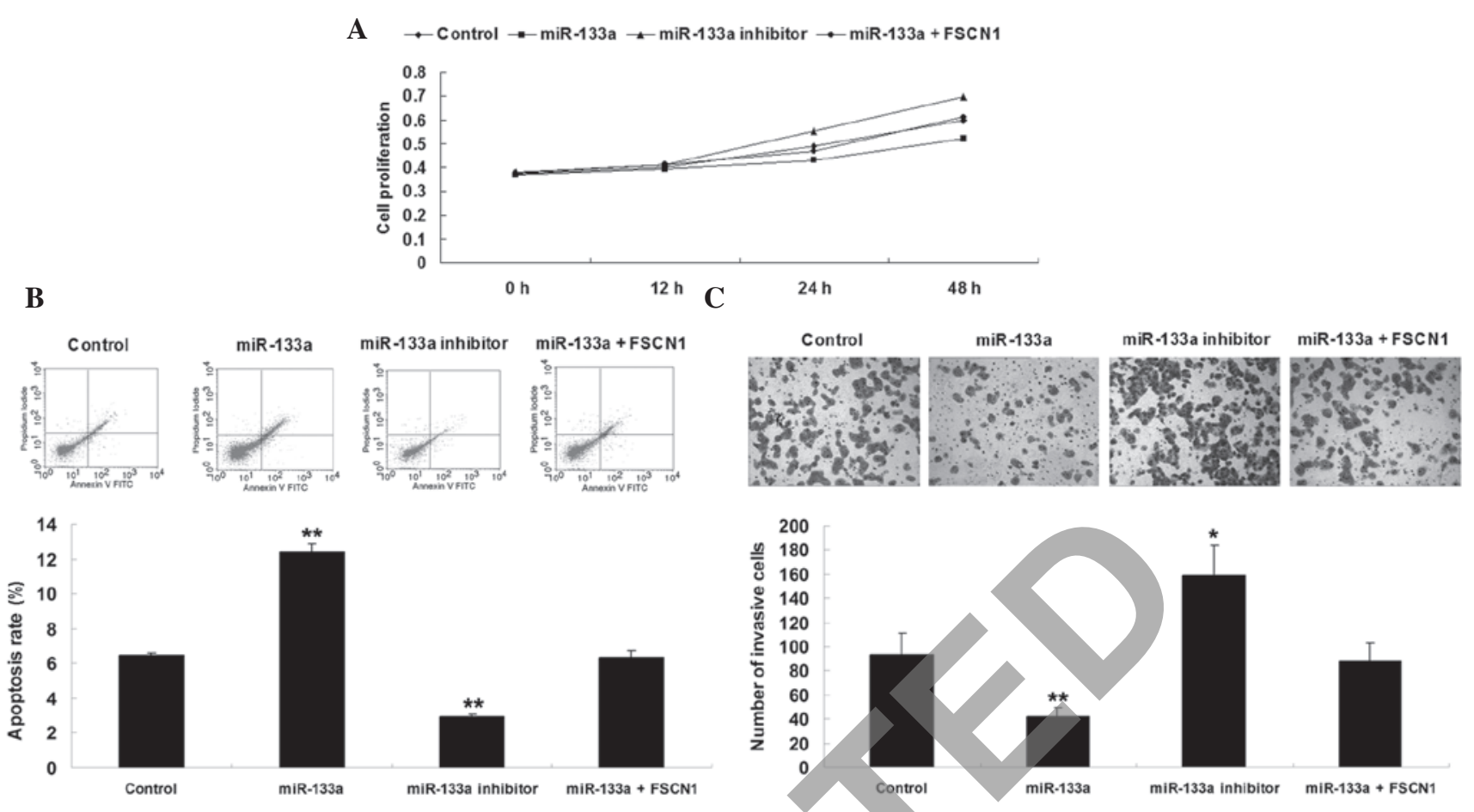

Figure 4. (A) An MTT assay (B) A cell apoptosis assay and (C) a Transwell assay were performed to examine the cell proliferation of AGS cells transfected with miR-133a mimics, miR-133a inhibitor, or co-transfected with miR-133a mimics and FSCN1 plasmid. Control: AGS cells without any transfection. ${ }^{*} \mathrm{P}<0.05$ vs. control. ${ }^{* *} \mathrm{P}<0.01$ vs. control. miR, microRNA; FSCN1, fascin actin-bundling protein 1.

was upregulated in gastric carcinoma tissues, compared with their matched normal adjacent tissues (Fig. 1C). In addition, the mRNA level of FSCN1 was also increased in gastric carcinoma cell lines, when compared with normal gastric epithelial cells (Fig. 1D). Accordingly, the present data suggested that miR-133a is downregulated while FSCN1 is upregulated in gastric carcinoma.

FSCN1 is a target of miR-133a in AGS gastric carcinoma cells. As shown in Fig. 2A, the putative seed sequences for miR-133a at the 3'UTR of FSCN1 was indicated based on bioinformatics analysis. To further confirm that FSCN1 is a target of miR-133a, the wild and mutant forms of the FSCN1 3'-UTR were generated (Fig. 2A). Subsequently, a luciferase reporter assay was performed using AGS gastric carcinoma cells. As shown in Fig. 2B, the luciferase activity was markedly decreased in AGS gastric carcinoma cells co-transfected with miR-133a mimics and the wild type 3'UTR of FSCN1, but unaltered in AGS cells co-transfected with miR-133a mimics and mutant FSCN1 3'UTR. The present data indicated that miR-133a FSCN1 is a target of miR-133a in AGS gastric carcinoma cells.

miR-133a negatively regulates the protein expression of FSCN1 in AGS gastric carcinoma cells. AGS cells were transfected with miR-133a mimics and inhibitor, and the protein expression of FSCN1 was determined in AGS cells. As shown in Fig. 3A, the transfection was efficient. It was then demonstrated that the protein level of FSCN1 was reduced following upregulation of miR-133a, but increased following inhibition of miR-133a (Fig. 3B). Therefore, it was suggested that miR-133a negatively regulates the protein expression of FSCN1 in AGS gastric carcinoma cells.
Effects of miR-133a and FSCN1 on AGS cell proliferation, apoptosis and invasion. At present, whether miR-133a is important in the regulation of cell proliferation, apoptosis, and invasion in gastric carcinoma cells via targeting FSCN1 remains to be elucidated. To investigate this mechanism, four groups were assigned. In the control group, AGS cells were cultured without transfection. In the miR-133a group, AGS cells were transfected with miR-133a mimics. In the miR-133a inhibitor group, AGS cells were transfected with miR-133a inhibitor. In the miR-133a + FSCN1 group, AGS cells were co-transfected with miR-133a mimics and FSCN1 plasmid. Subsequently, analysis of cell proliferation, apoptosis and invasion were performed. As shown in Fig. 4, overexpression of miR-133a inhibited proliferation and invasion, while promoted apoptosis in AGS cells, which was reversed by upregulation of FSCN1. By contrast, downregulation of miR-133a enhances proliferation and invasion, while suppressing apoptosis in gastric cancer cells. Accordingly, it was theorized that the role of miR-133a in the regulation of cell proliferation, apoptosis and invasion was, partly at least, through the inhibition of FSCN1.

\section{Discussion}

miRNAs are able to recognize target mRNAs based on complete or incomplete sequence complementarity and prevent their protein expression by binding to the 3 '-UTR of the target mRNA (5). In the present study, it was identified that FSCN1 is a target of miR-133a in AGS gastric cancer cells, and it was observed that FSCN1 was upregulated while miR-133a was downregulated in gastric cancer tissues and cell lines, when compared with normal gastric tissues and gastric epithelial cells. 
In addition, it was hypothesized that the effect of miR-133a on proliferation, apoptosis and invasion in AGS gastric cancer cells may be, partly at least, through the direct inhibition of FSCN1.

It has been well-established that deregulation of miRNAs is associated with the development and progression of cancer, including gastric cancer (6). In the present study, it was demonstrated that miR-133a was significantly downregulated in gastric carcinoma tissues and cell lines. In addition, Qiu et al (9) also revealed that the expression level of miR-133a was reduced in gastric carcinoma tissues and cell lines, consistent with the present findings. Furthermore, they identified that overexpression of miR-133a induced $G_{1}$ cell cycle arrest and inhibited cell proliferation, migration and invasion in gastric carcinoma cells (9). In the present study, it was also demonstrated that miR-133a upregulation inhibited cell proliferation and invasion in AGS gastric cancer cells. However, the molecular regulatory mechanism by which miR-133a regulates gastric cancer remains to be elucidated.

In the present study, it was identified that FSCN1 was involved in the effect of miR-133a on AGS cell proliferation, apoptosis and invasion. FSCN1 is a member of the FSCN family of actin-binding proteins. FSCN proteins are responsible for organization of F-actin into parallel bundles, and are involved in the formation of actin-based cellular protrusions. It has been well-established that FSCN1 is important in the regulation of cell adhesion motility and cellular interactions $(14,15)$. In addition, overexpression of FSCN1 has been observed to be associated with the metastasis of multiple types of cancer by increasing cell motility $(16,17)$. In addition, the deregulation of FSCN1 has been reported in gastric cancer. Tsai et al (18) revealed that among 60 samples of poorly-differentiated gastric adenocarcinomas, over half exhibited moderate or marked FSCN1 expression. Notably, a higher expression of FSCN1 correlated directly with more-advanced cancer stages and inversely with survival rates, suggesting that aberrant upregulation of FSCN1 may be involved in the progression of gastric adenocarcinomas. Fu et al (19) investigated the molecular mechanism by which FSCN1 may be involved in the regulation of gastric cancer. The authors revealed that FSCN1 is involved in TGF- $\beta 1$-induced gastric cancer cell invasion and metastasis. In addition, another study demonstrated that inhibition of FSCN1 expression suppressed the proliferation and metastasis of gastric cancer cells (20). In the present study, it was also observed that the expression of FSCN1 was upregulated in gastric cancer tissues. Based on these studies and the present data, it was hypothesized that FSCN1 may become a promising therapeutic target for gastric cancer.

Furthermore, the expression level of FSCN1 has been reported to be mediated by several microRNAs, including miR-145, miR-451 and miR-133b (21-23). In addition, the association between miR-133a and FSCN1 has been reported in several other types of cancer, including esophageal carcinoma, breast cancer and bladder cancer (13,24-26). For example, Akanuma et al (13) observed that the mRNA level of FSCN1 was upregulated in esophageal squamous cell carcinoma tissues and inversely correlated with the expression level of miR-133a. The authors further demonstrated that miR-133a inhibited cell proliferation and invasion, partly as least, via inhibition of FSCN1 in esophageal squamous cell carcinoma cells. Chiyomaru et al (24) revealed that miR-133a had a suppressive role through targeting FSCN1 in bladder cancer. In breast cancer, the expression of miR-133a was reduced in cancerous breast tissue compared with that of normal tissue and benign tumor tissue, and miR-133a was found to suppress tumor cell invasion and migration via targeting FSCN1 (26).

In conclusion, the present study indicated that the anti-oncogenic activity of miR-133a involved inhibition of the target gene FSCN1. The present study suggested that miR-133a may be a potential therapeutic target for gastric cancer.

\section{Acknowledgements}

The present study was supported by the Fundamental Research Funds for the Central Universities of Central South University (grant no. 2177-72150050583)

\section{References}

1. Piazuelo MB and Correa P: Gastric cancer: Overview. Colomb Med (Cali) 44: 192-201, 2013.

2. Jemal A, Bray F, Center MM, Ferlay J, Ward E and Forman D: Global cancer statistics. CA Cancer J Clin 61: 69-90, 2011.

3. Kuo CY, Chao Y and Li CP: Update on treatment of gastric cancer. J Chin Med Assoc 77: 345-353, 2014.

Piazuelo MB and Correa P: Gastric cancer: Overview. Colomb Med (Cali) 44: 192-201, 2013.

5. Ambros V: The functions of animal microRNAs. Nature 431: 350-355, 2004.

6. Li PF, Chen SC, Xia T, et al: Non-coding RNAs and gastric cancer. World J Gastroenterol 20: 5411-5419, 2014.

7. Bartel DP: MicroRNAs: genomics, biogenesis, mechanism and function. Cell 116: 281-297, 2004.

8. Hung $\mathrm{CH}$, Chiu $\mathrm{YC}$, Chen $\mathrm{CH}$ and $\mathrm{Hu} \mathrm{TH}$ : MicroRNAs in hepatocellular carcinoma: carcinogenesis, progression and therapeutic target. BioMed Res Int 2014: 486407, 2014.

9. Qiu T, Zhou X, Wang J, et al: MiR-145, miR-133a and miR-133b inhibit proliferation, migration, invasion and cell cycle progression via targeting transcription factor Sp1 in gastric cancer. FEBS Lett 588: 1168-1177, 2014.

10. Wang LK, Hsiao TH, Hong TM, et al: MicroRNA-133a suppresses multiple oncogenic membrane receptors and cell invasion in non-small cell lung carcinoma. PLoS One 9: e96765, 2014.

11. Guo J, Xia B, Meng F and Lou G: miR-133a suppresses ovarian cancer cell proliferation by directly targeting insulin-like growth factor 1 receptor. Tumour Biol 35: 1557-1564, 2014.

12. Fujiwara T, Katsuda T, Hagiwara K, et al: Clinical relevance and therapeutic significance of microRNA-133a expression profiles and functions in malignant osteosarcoma-initiating cells. Stem Cells 32: 959-973, 2014

13. Akanuma N,Hoshino I, Akutsu Y, et al: MicroRNA-133a regulates the mRNAs of two invadopodia-related proteins, FSCN1 and MMP14, in esophageal cancer. Br J Cancer 110: 189-198, 2014.

14. Jayo A and Parsons M: Fascin: a key regulator of cytoskeletal dynamics. Int J Biochem Cell Biol 42: 1614-1617, 2010.

15. Yang S, Huang FK, Huang J, et al: Molecular mechanism of fascin function in filopodial formation. J Biol Chem 288: 274-284, 2013.

16. Bi JB, Zhu Y, Chen XL, et al: The role of fascin in migration and invasion of urothelial carcinoma of the bladder. Urol Int 91: 227-235, 2013.

17. Tan VY, Lewis SJ, Adams JC and Martin RM: Association of fascin-1 with mortality, disease progression and metastasis in carcinomas: a systematic review and meta-analysis. BMC Med 11: $52,2013$.

18. Tsai WC, Jin JS, Chang WK, et al: Association of cortactin and fascin-1 expression in gastric adenocarcinoma: correlation with clinicopathological parameters. J Histochem Cytochem 55: 955-962, 2007.

19. Fu H, Hu Z, Wen J, Wang K and Liu Y: TGF-beta promotes invasion and metastasis of gastric cancer cells by increasing fascin1 expression via ERK and JNK signal pathways. Acta Biochim Biophys Sin (Shanghai) 41: 648-656, 2009.

20. Fu H, Wen JF, Hu ZL, Luo GQ and Ren HZ: Knockdown of fascin1 expression suppresses the proliferation and metastasis of gastric cancer cells. Pathology 41: 655-660, 2009. 
21. Chen MB, Wei MX, Han JY, et al: MicroRNA-451 regulates AMPK/mTORC1 signaling and fascin1 expression in HT-29 colorectal cancer. Cell Signal 26: 102-109, 2014

22. Adammek M, Greve B, Kässens N, et al: MicroRNA miR-145 inhibits proliferation, invasiveness and stem cell phenotype of an in vitro endometriosis model by targeting multiple cytoskeletal elements and pluripotency factors. Fertil Steril 99: 1346-1355 e5, 2013.

23. Yamamoto H, Kohashi K, Fujita A and Oda Y: Fascin-1 overexpression and miR-133b downregulation in the progression of gastrointestinal stromal tumor. Mod Pathol 26: 563-571, 2013.
24. Chiyomaru T,Enokida H, Tatarano S, et al: miR-145 and miR-133a function as tumour suppressors and directly regulate FSCN1 expression in bladder cancer. Br J Cancer 102: 883-891, 2010.

25. Kano M, Seki N, Kikkawa N, et al: miR-145, miR-133a and miR-133b: Tumor-suppressive miRNAs target FSCN1 in esophageal squamous cell carcinoma. Int J Cancer 127: 2804-2814, 2010.

26. Wu ZS, Wang CQ, Xiang R, et al: Loss of miR-133a expression associated with poor survival of breast cancer and restoration of miR-133a expression inhibited breast cancer cell growth and invasion. BMC Cancer 12: 51, 2012. 